\title{
GROWTH PROBLEMS FOR SUBHARMONIC FUNCTIONS OF FINITE ORDER IN SPACE
}

\author{
BY
}

N. V. RAO AND DANIEL F. SHEA(1)

\begin{abstract}
For a function $u(x)$ subharmonic (or $C^{2}$ ) in $\mathbf{R}^{m}$, we compare the "harmonics" (defined in \$1) of $u$ with those of a related subharmonic function whose total Riesz mass in $|x| \leqslant r$ is the same as that of $u$, but whose $L^{2}$ norm on $|x|=r$ is maximal, for all $0<r<\infty$. We deduce estimates on the growth of the Riesz mass of $u$ in $|x| \leqslant r$, as $r \rightarrow \infty$.
\end{abstract}

Introduction. Following Hayman [7], [8], we study the growth and distribution of the Riesz mass of subharmonic functions in $\mathbf{R}^{m}(m \geqslant 2)$ from the point of view of classical value distribution theory. Thus, if $u(x)$ is subharmonic we define the characteristic

$$
T(r, u)=\sigma_{m}^{-1} \int_{|\omega|=1} u(r \omega)^{+} d \omega
$$

of $u(x)$ and its order

$$
\lambda=\limsup _{r \rightarrow \infty} \frac{\log T(r, u)}{\log r}
$$

$d \omega$ denotes $(m-1)$-dimensional surface area on $\Sigma=\Sigma_{m}=\{|x|=1\}$ and $\sigma_{m}=\int_{\Sigma} d \omega$. We always suppose $u^{+}$is unbounded: $T(r, u) \rightarrow \infty$ when $r \rightarrow \infty$, and $u$ is harmonic near 0 with $u(0)=0$. We compare the growth of $T(r, u)$ with that of

$$
N(r)=N_{u}(r)=\sigma_{m}^{-1} \int_{\Sigma} u(r \omega) d \omega
$$

which, by Jensen's theorem [1, p. 133], is a weighted average of the Riesz mass of $u$ in the ball $|x| \leqslant r$ :

Presented to the Society, January 23, 1975; received by the editors January 13, 1976. AMS (MOS) subject classifications (1970). Primary 30A70, 31B05; Secondary 32H25.

(') The authors were supported by NSF Grants GP-33897X and GP-21340.

- American Mathematical Society 1977 
(4) $\quad n(r)=\left(\sigma_{m} d_{m}\right)^{-1} \int_{|x| \leqslant r} d(\Delta u(x)), \quad N(r)=d_{m} \int_{0}^{r} n(t) t^{1-m} d t$.

Here $\Delta$ denotes the Laplacian, $\Delta u$ exists as a distribution and $\mu=\left(\sigma_{m} d_{m}\right)^{-1} \Delta u$ is a positive measure when $u$ is subharmonic [1, p. 127]; and $d_{m}=m-2$ for $m>2, d_{2}=1$. (For definitions and a discussion of basic results, see $\S 1$.)

When $f(z)$ is an entire function of one complex variable and $u(x, y)$ $=\log |f(x+i y)|, n(r)$ counts the number of zeros of $f(z)$ in $|z| \leqslant r$, and it is a classical problem to find good lower bounds for

$$
k(u)=\limsup _{r \rightarrow \infty} \frac{N(r)}{T(r, u)}
$$

in terms of $\lambda$. For example, it is known in this case that

$$
k(u) \geqslant \begin{cases}1 & \left(0 \leqslant \lambda \leqslant \frac{1}{2}\right) \\ \sin \pi \lambda & \left(\frac{1}{2}<\lambda \leqslant 1\right)\end{cases}
$$

(Edrei and Fuchs [3]), where equality holds for $f(z)=$ polynomial $(\lambda=0)$, $=e^{z}(\lambda=1)$ and

$$
f(z)=\prod_{n=1}^{\infty}\left(1-z / n^{1 / \lambda}\right) \quad(0<\lambda<1) .
$$

Hayman has extended (6) to arbitrary subharmonic $u$ in the plane and found the sharp analogue for functions of orders $\lambda<1$ in $\mathbf{R}^{m}, m \geqslant 3$ ([7], [8]).

For $\lambda>1$, precise results are not in general available even for entire functions. A recent result in this direction is

$$
k(u) \geqslant(0.9) \frac{|\sin \pi \lambda|}{\lambda+1} \quad(1<\lambda<\infty)
$$

(Miles and Shea [10]), and well-known examples [2] show that (8) would fail for large $\lambda$ if the 0.9 factor were replaced by any constant greater than unity. Inequality (8) is an easy corollary of the main result of [10],

THEOREM A. Let $f(z)$ be an entire function of finite order $\lambda$ in the plane, and put $u(z)=\log |f(z)|$,

$$
m_{2}(r, u)=\left\{\frac{1}{2 \pi} \int_{0}^{2 \pi}\left|u\left(r e^{i \theta}\right)\right|^{2} d \theta\right\}^{1 / 2}
$$

Then

$$
\underset{r \rightarrow \infty}{\lim \sup _{2}} \frac{N(r)}{m_{2}(r, u)} \geqslant \frac{|\sin \pi \lambda|}{\pi \lambda}\left\{\frac{2}{1+(\sin 2 \pi \lambda) / 2 \pi \lambda}\right\}^{1 / 2} .
$$


Equality is possible in (10) for each $\lambda \geqslant 0$.

Our first purpose in this note is to find the appropriate extension of Theorem A to subharmonic functions. The proof in [10] rests on some simple properties of the Fourier coefficients

$$
c_{k}(r ; f)=\frac{1}{2 \pi} \int^{2 \pi} \log \left|f\left(r e^{i \theta}\right)\right| e^{-i k \theta} d \theta \quad(k=0, \pm 1, \pm 2, \ldots),
$$

in particular on the inequality

$$
\left|c_{k}(r ; f)\right| \leqslant\left|c_{k}\left(r ; f^{*}\right)\right| \quad(r>0, k=0, \pm 1, \pm 2, \ldots)
$$

where $f^{*}$ is a suitable entire function whose zeros have the same moduli as those of $f$ but are projected onto the positive real axis. Thus, if $u^{*}=\log \left|f^{*}\right|$, then $N_{u}(r) \equiv N_{u^{*}}(r)$ and

$$
m_{2}(r, u) \leqslant m_{2}\left(r, u^{*}\right) \quad(0<r<\infty)
$$

by Parseval's theorem, and to prove (10) it suffices to consider just the $f^{*}$.

In $\$ 2$, we study the spherical harmonics of subharmonic functions in $\mathbf{R}^{\boldsymbol{m}}$ and prove an analogue of (11) for all $m \geqslant 2$ (Theorem 2.1). From this we deduce

THEOREM 1. Let $u(x)$ be subharmonic and of finite order $\lambda$ in $\mathbf{R}^{m}$, and put

$$
m_{2}(r, u)=\left\{\sigma_{m}^{-1} \int_{\Sigma}|u(r \omega)|^{2} d \omega\right\}^{1 / 2}
$$

Then

$$
\underset{r \rightarrow \infty}{\limsup } \frac{N(r)}{m_{2}(r, u)} \geqslant C(\lambda, m) \quad(0 \leqslant \lambda<\infty, m \geqslant 2),
$$

where

$$
C(\lambda, m)=\left\{1+\frac{\lambda^{2}(\lambda+m-2)^{2}}{(m-2) !} \sum_{k=1}^{\infty} \frac{(k+m-3) !(2 k+m-2)}{k !(k-\lambda)^{2}(k+\lambda+m-2)^{2}}\right\}^{-1 / 2}
$$

When $m=2$, the bound in (13) is the same as that in (10), and when $m=3$ or 4 inequality (13) remains sharp for all $\lambda$, with 


$$
\begin{aligned}
& C(\lambda, 3)=\frac{|\sin \pi \lambda| \sqrt{2 \lambda+1}}{\pi \lambda(\lambda+1)}\left\{1-\frac{2}{\pi^{2}}\left(\sin ^{2} \pi \lambda\right) \sum_{k=1}^{\infty} \frac{1}{(k+\lambda)^{2}}\right\}^{-1 / 2}, \\
& C(\lambda, 4)=\frac{|\sin \pi \lambda|}{\pi \lambda\left(\frac{1}{2} \lambda+1\right)}\left\{1-\frac{\sin 2 \pi \lambda}{2 \pi(\lambda+1)}\right\}^{-1 / 2} .
\end{aligned}
$$

When $m \geqslant 5$ the series in (14) diverges and $C(\lambda, m) \equiv 0$, which just reflects the fact that for these $m$ the extremal functions for this problem (studied in §4) fail to be square-integrable on spheres $|x|=r, 0<r<\infty$.

By Schwarz's inequality and Jensen's theorem, $m_{2}(r, u) \geqslant 2 T(r, u)-N(r)$, and we deduce easily a bound for $k(u)$ defined in (5):

COROLLARY 1. If $u(x)$ is subharmonic

$$
k(u) \geqslant \frac{|\sin \pi \lambda|}{\pi \lambda(\lambda+1)^{\frac{1}{2} m-1}} \quad(0 \leqslant \lambda<\infty ; m=2,3,4) .
$$

In $\S 4$ we consider a class of examples which, we conjecture, minimize $k(u)$ for any given order $\lambda$ and dimension $m$; in particular we show that there exist subharmonic functions $u_{\lambda, m}(x)$ of order $\lambda$ in $\mathbf{R}^{m}$ with

$$
k\left(u_{\lambda, m}\right) \leqslant C_{m} \frac{|\sin \pi \lambda|}{(\lambda+1)^{\frac{1}{2} m}} \quad(1<\lambda<\infty) .
$$

Thus the bound in (15) has the right order of magnitude for large $\lambda$.

Using other methods, we obtain

THEOREM 2. If $u(x)$ is subharmonic and of order $\lambda$ in $\mathbf{R}^{m}$, then

$$
k(u) \geqslant A_{m} \frac{|\sin \pi \lambda|}{(\lambda+1)^{\frac{1}{2}(m+1)}} \quad(0<\lambda<\infty ; m \geqslant 5)
$$

where $A_{m}$ depends only on $m$.

Hayman [8] has obtained $k(u) \geqslant(q+1-\lambda)(\lambda-q) / \lambda(q+1) 4^{m+q}$, with $q=[\lambda]$, as a consequence of an inequality between $N(r)$ and $M(r, u)$ $=\sup _{|x|=r} u(x)$. Using the Poisson formula to estimate $M(r, u)$ in terms of $T(\sigma r, u), \sigma>1$, we can easily adapt the proof of (17) to find that

$$
\limsup _{r \rightarrow \infty} \frac{N(r)}{M(r, u)} \geqslant B_{m} \frac{|\sin \pi \lambda|}{(\lambda+1)^{\frac{3}{2} m}} \quad(0<\lambda<\infty, m \geqslant 2) .
$$

The conjectured extremal functions $u_{\lambda, m}$ mentioned above are harmonic in $\mathbf{R}^{m}$ except on the positive $x_{1}$-axis, along which the Riesz mass is distributed 
regularly: $N_{u_{\lambda, m}}(r) \equiv r^{\lambda}$, and $u_{\lambda, m}(x)=|x|^{\lambda} I(\cos \theta ; \lambda, m)$ where $\theta$ denotes the angle between the vector $x$ and the positive $x_{1}$-axis, and $I$ is defined in $\S 4$. If we put

$$
K(\lambda, m) \stackrel{\text { def }}{=} k\left(u_{\lambda, m}\right)=T\left(1, u_{\lambda, m}\right)^{-1} \quad(m \geqslant 2,0 \leqslant \lambda<\infty)
$$

then Hayman's sharp result noted earlier, for $\lambda<1$ and $m \geqslant 2$, is: $k(u)$ $\geqslant K(\lambda, m)$, and our approximations (15) and (17) for $\lambda>1$ have been compared with $K(\lambda, m)$ via (16). Complementary to these lower bounds for $k(u)$, when $u$ is an arbitrary subharmonic function, is

THEOREM 3. Let $u(x)$ be subharmonic in $\mathbf{R}^{m}$ of finite nonintegral order $\lambda$ with all its Riesz mass distributed along a ray through 0 . Then

$$
\liminf _{r \rightarrow \infty} \frac{N(r)}{T(r, u)} \leqslant K(\lambda, m)
$$

where besides (16) $K(\lambda, m)$ satisfies

$$
K(\lambda, m)<1 \quad(m \geqslant 3,0<\lambda<\infty)
$$

and

$$
\begin{aligned}
K(\lambda, 2) & =\frac{|\sin \pi \lambda|}{q+|\sin \pi \lambda|} \quad\left(q \leqslant \lambda<q+\frac{1}{2}\right) \\
& =\frac{|\sin \pi \lambda|}{q+1} \quad\left(q+\frac{1}{2} \leqslant \lambda<1\right)
\end{aligned}
$$

for $q=0,1,2, \ldots$

Inequality (18) remains valid for integral orders $\lambda$, but then requires different methods; cf. [15].

For entire functions in the plane this is due to Ostrovskii [12]. There exist other related studies of this type, e.g. by Edrei and Fuchs [2], also [4], [5], [9].

All the results mentioned above for entire functions have extensions to meromorphic functions, provided the definitions of $N(r)$ and $T(r, u)$ are generalized in a natural way. If $f$ is meromorphic in the plane and $u(z)$ $=\log |f(z)|=\log |g(z)|-\log |h(z)|$ where $g, h$ are entire functions having no common zeros, we define $\mu=\Delta u=\Delta \log |g|-\Delta \log |h|=\mu^{+}-\mu^{-}$where $\mu^{+}$ and $\mu^{-}$are positive measures,

$$
\begin{aligned}
n(r, u) & =\frac{1}{2 \pi} \int_{|z| \leqslant r} d \mu^{-}(z), & n(r,-u) & =\frac{1}{2 \pi} \int_{|z| \leqslant r} d \mu^{+}(z), \\
N(r, u) & =\int_{0}^{r} n(t, u) t^{-1} d t, & N(r) & =N_{u}(r)=N(r, u)+N(r,-u),
\end{aligned}
$$

with 


$$
T(r, u)=\frac{1}{2 \pi} \int_{0}^{2 \pi} u\left(r e^{i \theta}\right)^{+} d \theta+N(r, u), \quad k(u)=\limsup _{r \rightarrow \infty} \frac{N(r)}{T(r, u)}
$$

Thus $k(u)$ gives a measure of the "total deviation from harmonicity" of $u=\log |f|$. The Edrei-Fuchs inequality (6) remains valid in this more general setting [3], as does Theorem A [10].

We prove Theorems 1 and 2 for $u(x)$ in the class $\mathscr{D}_{m}$ of functions "deltasubharmonic" in $\mathbf{R}^{m}$.

Definition 1. A function $u$ defined (a.e.) in $\mathbf{R}^{m}$ is in $\mathscr{D}_{m}$ if there exist subharmonic functions $u_{1}, u_{2}$ in $\mathbf{R}^{m}$ with $u=u_{1}-u_{2}$.

A more intrinsic definition is: $u \in \mathscr{D}_{m}$ if for every compact set $F, u$ $\in L^{1}(F)$ and

$$
\left|\int u(x) \Delta \varphi(x) d x\right| \leqslant C(F)\|\varphi\|_{\infty}
$$

for some constant $C(F)$ and every $\varphi \in C^{\infty}\left(\mathbf{R}^{m}\right)$ vanishing outside of $F$.

It is immediate from the second definition that any $u \in C^{2}\left(\mathbf{R}^{m}\right)$ is deltasubharmonic. The equivalence of the two definitions and other basic facts needed here are discussed further in $\$ 1$.

If $f: \mathbf{C}^{M} \rightarrow \mathbf{C}$ is an entire function of order $\lambda$, then Theorem 2 applies to $u=\log |f|$ and yields

$$
\limsup _{r \rightarrow \infty} \frac{N(r, 0 ; f)}{T(r, f)} \geqslant A(M) \frac{|\sin \pi \lambda|}{\lambda^{c}+1} \quad(0<\lambda<\infty)
$$

with $c=M+\frac{1}{2}$ and $N(r, 0 ; f) \equiv N_{u}(r)$. Our examples $u_{\lambda, 2 M}(x)$ show that $c \geqslant M$ for subharmonic functions in $\mathbf{R}^{2 M}$ generally, but it remains an interesting question whether (22) with $c \approx M$ is a good estimate for entire functions when $M \geqslant 2$.

1. Definitions and auxiliary results. A function $u: \mathbf{R}^{m} \rightarrow[-\infty, \infty)$ is subharmonic, $u \in \delta_{m}$, if $u$ is upper semicontinuous, $\not \equiv-\infty$ and

$$
u(x) \leqslant \sigma_{m}^{-1} \int_{\Sigma} u(x+\delta \omega) d \omega
$$

for all $x \in \mathbf{R}^{m}$ and $\delta>0$. It is well known [1, p. 128], [8], [14] that

$$
u \in L^{1}(F) \text { for every compact } F \text {, }
$$

$\Delta u$ exists as a distribution and $\mu=\left(\sigma_{m} d_{m}\right)^{-1} \Delta u$

is a positive Borel measure, finite for compact sets.

Further, Riesz's theorem holds: If 


$$
K(x)=\log |x| \quad(m=2), \quad=-|x|^{2-m} \quad(m \geqslant 3),
$$

then for any compact $F$,

$$
u(x)=\int_{F} K(x-y) d \mu(y)+h(x)
$$

where $\mu$ is the measure in (1.2) and $h$ is harmonic in the interior of $F$. Conversely, given a positive locally finite measure $\mu$ on $\mathbf{R}^{m}$, any $u$ having the representation (1.4) for compact $F$ and $h$ harmonic in the interior of $F$ is subharmonic in $\mathbf{R}^{m}$ with $\Delta u=\sigma_{m} d_{m} \mu$.

The measure $\mu$ in (1.2) is termed the Riesz measure of $u$.

Let $u \in \mathscr{D}_{m}$, so that $u=u_{1}-u_{2}$ where $u_{j} \in \mathrm{S}_{m}$. Then it is clear that (21) holds with $C(F)=\mu_{1}(F)+\mu_{2}(F)$ if $\mu_{j}=\Delta u_{j}$ for $j=1,2$. Conversely, suppose $u \in L_{\text {loc }}^{1}$ satisfies (21). Then $\Delta u$ is a (locally finite, signed) Borel measure $\sigma_{m} d_{m} \mu[1$, p. 93]. Let $|\mu|$ be the total variation of $\mu$, and let $\mu^{+}=\frac{1}{2}(|\mu|+\mu), \mu^{-}=\frac{1}{2}(|\mu|-\mu)$. Then as in Weierstrass's classical theorem we can construct [8, Chapter 4] functions $u^{+}, u^{-} \in \delta_{m}$ with $\Delta u^{ \pm}=\sigma_{m} d_{m} \mu^{ \pm}$ and $u=u^{+}-u^{-}+h$ where $h$ is harmonic in $\mathbf{R}^{m}$; thus $u \in \mathscr{D}_{m}$ according to Definition 1.

For convenience, we shall continue to refer to the measure defined in (1.2) as the Riesz measure of $u$, for any $u \in \mathscr{D}_{m}$, and to the mass of the total variation measure $|\mu|=\mu^{+}+\mu^{-}$as the Riesz mass of $u$.

If $u \in \mathscr{D}_{m}$,

$$
\mu=\left(\sigma_{m} d_{m}\right)^{-1} \Delta u=\mu^{+}-\mu^{-}
$$

and we assume throughout $\S \S 1-3$ that $\mu^{+}, \mu^{-}$have no mass in a neighborhood of 0 , that

and that

$$
u(0)=0 \text {, }
$$

This involves no restriction for the kind of asymptotic problems studied here.

Generalizing definitions (1), (4) we put

$$
\begin{aligned}
n(r, u) & =\mu^{-}(\{|x| \leqslant r\}), \quad n(r,-u)=\mu^{+}(\{|x| \leqslant r\}), \\
N(r, u) & =d_{m} \int_{0}^{r} n(t, u) t^{1-m} d t, \\
N(r) & =N_{u}(r)=N(r, u)+N(r,-u), \\
T(r, u) & =\sigma_{m}^{-1} \int_{\Sigma} u^{+}(r \omega) d \omega+N(r, u),
\end{aligned}
$$

and (2), (5) remain unchanged. 
Applying Green's formula to $u_{2}$, we have

$$
u_{2}(0)=\sigma_{m}^{-1} \int_{\Sigma} u_{2}(r \omega) d \omega+\int_{|y| \leqslant r}[K(y)-K(r e)] d \mu^{-}(y)
$$

where $e=(1,0, \ldots, 0)$, and integration by parts converts the last integral in (1.9) to $N(r, u)$. Thus

$$
\begin{aligned}
T(r, u) & =\sigma_{m}^{-1} \int_{\Sigma}\left[\left(u_{1}-u_{2}\right)^{+}+u_{2}\right](r \omega) d \omega-u_{2}(0) \\
& =\sigma_{m}^{-1} \int_{\Sigma} v(r \omega) d \omega-u_{2}(0)
\end{aligned}
$$

where $v=\max \left(u_{1}, u_{2}\right) \in \delta_{m}$, so that by (3) and (4), $T(r, u)$ is a continuous, increasing function convex in $\log r(m=2), r^{2-m}(m \geqslant 3)$.

Applying (1.9) to $u$, we obtain the analogue for $u \in \mathscr{D}_{m}$ of Nevanlinna's first fundamental theorem,

$$
T(r, u)=T(r,-u) \quad(0<r<\infty) .
$$

If $x, y \in \mathbf{R}^{m}$ we write

$$
x \vee y=x \cdot y /|x||y|=\cos \theta
$$

where $\theta$ is the angle between $\overrightarrow{0 x}$ and $\overrightarrow{0 y}$. Then

$$
\begin{aligned}
K(x-y) & =-\sum_{k=0}^{\infty} P_{k}(x \vee y) \frac{|x|^{k}}{|y|^{k+m-2}} & & (|x|<|y|) \\
& =-\sum_{k=0}^{\infty} P_{k}(x \vee y) \frac{|y|^{k}}{|x|^{k+m-2}} & & (|y|<|x|)
\end{aligned}
$$

where the $P_{k}$ are the Gegenbauer polynomials [16, pp. 302, 329]. On the other hand, for fixed $y, K(x-y)$ is real-analytic in $x$ and thus $P_{k}(x \vee y)$ $\cdot|x|^{k} /|y|^{k+m-2}$ is the sum of terms of degree $k$ in the Taylor expansion of $K(x-y)$ in a neighborhood of the origin. Thus $P_{k}(x \vee y)|x|^{k}$ is a homogeneous harmonic polynomial of degree $k$ in $x$ (except when $m=2, k=0$ ), and $[1$, p. 169]

$$
\int_{\Sigma} P_{j}(r \omega \vee y) P_{k}(r \omega \vee z) d \omega=0 \quad(j \neq k)
$$

for all $r=|x|>0$ and $y, z \in \mathbf{R}^{m}-\{0\}$.

For any integer $q \geqslant 0$, we define

$$
K_{q}(x, y)=K(x-y)+\sum_{k=0}^{q} P_{k}(x \vee y) \frac{|x|^{k}}{|y|^{k+m-2}} \quad\left(x, y \in \mathbf{R}^{m}\right) .
$$

Thus 


$$
K_{q}(x, y)=-\sum_{k=q+1}^{\infty} P_{k}(x \vee y) \frac{|x|^{k}}{|y|^{k+m-2}} \quad(|x|<|y|) .
$$

Assume that $u \in \mathscr{D}_{m}$ is of finite order $\lambda$, so that by (1.8) and (1.10):

$$
\limsup _{r \rightarrow \infty} \frac{\log N(r)}{\log r} \leqslant \lambda,
$$

and let $\mu$ be the associated Riesz measure. Then for $\alpha>\lambda$,

$$
\begin{aligned}
\int_{0}^{\infty} \frac{N(r)}{r^{\alpha+1}} d r & =\frac{d_{m}}{\alpha} \int_{0}^{\infty} \frac{n(t)}{t^{\alpha+m-1}} d t \\
& =\frac{d_{m}}{\alpha(\alpha+m-2)} \int_{\mathbf{R}^{m}} \frac{d|\mu|(x)}{|x|^{\alpha+m-2}}
\end{aligned}
$$

converges. Let $q=q(\mu)$ denote the least integer $\geqslant 0$ for which

$$
\int \frac{d|\mu|(x)}{|x|^{q+m-1}}<\infty
$$

and put

$$
u_{\mu}(x)=\int_{\mathbf{R}^{m}} K_{q}(x, y) d \mu(y) .
$$

By (1.16), (1.13), (1.11) and (1.4), $u_{\mu} \in \mathscr{D}_{m}$ and

$$
u_{\mu}(r \omega) \in L^{1}(\Sigma, d \omega) \quad(0<r<\infty) .
$$

For some purposes it is convenient to have explicit estimates of $K_{q}$, and we state

LEMmA 1.1. There exists a constant $C=C(m, q)$ such that, if $|x|=r$,

$$
\begin{aligned}
\left|K_{q}(x, y)\right| & \leqslant C r^{q+1} /|y|^{q+m-1} \quad\left(r \leqslant \frac{1}{2}|y|\right), \\
K_{q}(x, y) & \leqslant C r^{q+1} /|y|^{q+m-2}(r+|y|) \quad\left(x, y \in \mathbf{R}^{m}\right),
\end{aligned}
$$

the latter except when $m=2$ and $q=0$, in which case

$$
K_{0}(x, y)=\log |1-x / y| \leqslant \log (1+r /|y|) .
$$

When $m=2$, Lemma 1.1 is well known [6, p. 26]; analogous estimates yield the result for $m \geqslant 3$, e.g. see [8].

Using Lemma 1.1 we find that if 


$$
\lambda_{0}=\limsup _{r \rightarrow \infty} \frac{\log N(r)}{\log r},
$$

then $u_{\mu}$ has order $\lambda_{0}, q \leqslant \lambda_{0} \leqslant q+1$. Further, arguments like those used for the classical Hadamard representation theorem (worked out in Hayman's book [8, Chapter IV]), give

LEMMA 1.2. Let $u \in \Phi_{m}$ have finite order $\lambda$, let $q(\mu)$ be determined as in (1.16) and put $g=\max (q,[\lambda])$.

Then

$$
u(x)=u_{\mu}(x)+h(x)
$$

where $h$ is a harmonic polynomial of degree at most $g$.

Observe that $g=q(\mu)$ when $\lambda$ is not a positive integer.

Finally, we collect some facts about spherical harmonics needed for Theorem 1; for proofs see [1, pp. 168-170] and [11, pp. 43, 44]. Let $\mathcal{K}_{k}$ denote the space of all homogeneous harmonic polynomials of degree $k$. The restrictions of these to $\Sigma$ are the spherical harmonics of order $k$, and they form a finite-dimensional subspace $\varrho_{k}$ of $L^{2}(\Sigma, d \omega)$. For each $k \geqslant 0$, let $\left\{\varphi_{k, j}\right\}_{j=0}^{n(k)}$ be an orthonormal basis of $\complement_{k}$; then the set $\Phi=\left\{\varphi_{k, j}: k \geqslant 0,0 \leqslant j \leqslant n(k)\right\}$ is complete in $L^{2}(d \omega)$. If $\varphi, \psi \in \Phi$ are of different degrees then $\int_{\Sigma} \varphi(\omega) \psi(\omega) d \omega$ $=0$; this fact generalizes (1.12).

Let $f \in L^{1}(d \omega)$, and define the $k$ th harmonic of $f$ to be

$$
f_{k}=\sum_{j=0}^{n(k)}\left\{\int_{\Sigma} f(\omega) \varphi_{k, j}(\omega) d \omega\right\} \varphi_{k, j}
$$

We note that

$$
\left\|f_{k}\right\|_{\infty} \leqslant C(k)\|f\|_{1} \quad(k \geqslant 0),
$$

that $f=\sum f_{k}$ holds for all $f$ in the linear span $\Phi^{*}$ of $\Phi$, and that if $f_{k} \equiv 0$ for each $k \geqslant 0$ then $f \equiv 0$, since $\Phi^{*}$ is dense in $C(\Sigma)$. Further, $f_{k}$ is the orthogonal projection of $f$ onto $\mathfrak{L}_{k}$ for all $f \in L^{2}(d \omega)$, and thus $f_{k}$ does not depend on the basis chosen.

Finally, we write

$$
c_{k}=c_{k}(f)=\left\{\int_{\Sigma} f_{k}^{2}(\omega) d \omega\right\}^{1 / 2}=\left\|f_{k}\right\|_{2}
$$

and observe that, if $f \in L^{2}(d \omega)$,

$$
\|f\|_{2}=\left\{\sum_{k=0}^{\infty} c_{k}^{2}\right\}^{1 / 2}
$$

since $\Phi$ is complete. 
In the next section we study the harmonics of $u_{\mu}$ defined in (1.17), and for this we must compute the harmonics of $K_{q}$. For a given $r>0$ and $y \in \mathbf{R}^{m}$, let $\left\{\varphi_{k, j}\right\}_{j=0}^{n(k)}$ be as described above with $\varphi_{k, 0}(\omega)=\alpha_{k} P_{k}(\omega \vee y)$, where the positive number $\alpha_{k}$ is determined by $\left\|\varphi_{k, 0}\right\|_{2}=1$. Then it is obvious from (1.12)-(1.14) that the $k$ th harmonic of $f(\omega)=K_{q}(r \omega, y)$ is

$$
f_{k}(\omega)=Q_{k} P_{k}(\omega \vee y) \quad(\omega \in \Sigma)
$$

for a suitable factor $Q_{k}(r,|y|)$. When $|y|>r$ we compute $Q_{k}$ using (1.14),

$$
Q_{k}(r,|y|)=-r^{k} /|y|^{k+m-2} \quad(k>q), \quad=0 \quad(k \leqslant q) .
$$

When $|y|<r$ we use (1.13) in a similar way and, for $|y|=r, Q_{k}$ is defined by continuity since $K_{q}(r \omega, \sigma y) \rightarrow K_{q}(r \omega, y)$ in $L^{1}(d \omega)$ when $\sigma \rightarrow 1$. Then the values of $Q_{k}$ can be tabulated as follows:

\begin{tabular}{|c|c|c|}
\hline$Q_{k}(r, t)$ & $t<r$ & $t \geqslant r$ \\
\hline$k>q$ & $-t^{k} / r^{k+m-2}$ & $-r^{k} / t^{k+m-2}$ \\
$1 \leqslant k \leqslant q$ & $r^{k} / t^{k+m-2}-t^{k} / r^{k+m-2}$ & 0 \\
$k=0$ & $K(r)-K(t)$ & 0 \\
\hline
\end{tabular}

Finally, we observe from (1.17) and Fubini's theorem that the $k$ th harmonic of $u_{\mu}(r \omega)$ is

$$
\int_{\mathbf{R}^{m}} Q_{k}(r,|y|) P_{k}(\omega \vee y) d \mu(y)
$$

2. An extremal property of spherical symmetrizations of potentials; proof of Theorem 1. Let $u \in \mathscr{D}_{m}$ have finite nonintegral order $\lambda$, and let $q=[\lambda]$. Let $\mu$ be the Riesz measure of $u$, and denote by $\tilde{\mu}$ the measure obtained by projecting the mass of $\mu$ onto the positive $x_{1}$-axis according to

$$
\tilde{\mu}([a, b])=\mu(\{a \leqslant|x| \leqslant b\}) \quad(0<a<b<\infty)
$$

where $[a, b]$ denotes the interval on the $x_{1}$-axis with endpoints $(a, 0, \ldots, 0)$, $(b, 0, \ldots, 0)$. We also introduce the total variation measure $\mu^{*}=|\tilde{\mu}|$ and the associated subharmonic function

$$
u_{\mu}^{*}(x)=\int_{0}^{\infty} K_{q}(x, t e) d \mu^{*}(t)
$$

We shall compare the harmonics of $u_{\mu}$ and $u_{\mu}^{*}$. Recalling (1.22) and (1.25) we define

$$
C_{k}\left(r, u_{\mu}\right)=c_{k}\left(u_{\mu}(r \omega)\right)=\left\|\int_{\mathbf{R}^{m}} Q_{k}(r,|y|) P_{k}(\omega \vee y) d \mu(y)\right\|_{2} .
$$


If $u_{\mu}(r \omega) \in L^{2}(d \omega), m_{2}\left(r, u_{\mu}\right)$ defined in Theorem 1 satisfies

$$
m_{2}\left(r, u_{\mu}\right)=\sigma_{m}^{-1 / 2}\left\|u_{\mu}\right\|_{2}=\left\{\sigma_{m}^{-1} \sum_{k=0}^{\infty} C_{k}\left(r, u_{\mu}\right)^{2}\right\}^{1 / 2},
$$

see (1.23). In any case, we have

THEOREM 2.1. Let $\mu$ be a measure satisfying (1.16). Then

$$
C_{k}\left(r, u_{\mu}\right) \leqslant C_{k}\left(r, u_{\mu}^{*}\right) \quad(0<r<\infty ; k \geqslant 0) .
$$

Thus

$$
m_{2}\left(r, u_{\mu}\right) \leqslant m_{2}\left(r, u_{\mu}^{*}\right)
$$

for all $r$ such that $m_{2}\left(r, u_{\mu}^{*}\right)<\infty$. [This holds everywhere when $m=2$, and a.e. when $m=3$, 4; for, by (2.1) it is sufficient to show, a.e.,

$$
\psi_{r}(\omega)=\int_{r / 2}^{2 r}|r \omega-t e|^{2-m} d \mu^{*}(t) \in L^{2}(d \omega) .
$$

Fix any $r$ such that $\varphi(t)=\mu^{*}\{[0, t]\}$ has a finite derivative at $r$, and let $\delta$ and $K$ satisfy $|\varphi(t)-\varphi(r)| \leqslant K|t-r|$ when $|t-r| \leqslant 2 \delta$. Then

$$
\begin{aligned}
& \psi_{r}(\cos \theta)= \int_{r / 2}^{2 r}\left\{t^{2}+r^{2}-2 t r \cos \theta\right\}^{-\nu} d \varphi(t) \quad\left(\nu=\frac{1}{2}(m-2)\right) \\
& \leqslant C \int_{r / 2}^{2 r}\{|r-t|+\theta\}^{-2 v} d \varphi(t) \\
& \leqslant C\left\{\int_{|r-t| \leqslant \theta} \theta^{-2 v} d \varphi(t)+\sum_{j=0}^{k} \int_{2^{j} \theta<|r-t| \leqslant 2^{j+1} \theta}|r-t|^{-2 v} d \varphi(t)\right. \\
& \\
&\left.+\int_{\delta<|r-t| \leqslant r}|r-t|^{-2 v} d \varphi(t)\right\}
\end{aligned}
$$

where $C$ depends only on $r$ and $k=[\log (\delta / \theta) / \log 2]$. It follows that $\psi_{r}(\cos \theta)$ $\in L^{2}\left([0, \pi] ; \sin ^{m-2} \theta d \theta\right)$ when $m=3,4$.]

ProOF OF THEOREM 2.1. For each $k>0$ we have by Schwarz's inequality and the fact that, by (1.24), $Q_{k}$ is of one sign only,

$$
\begin{aligned}
C_{k}\left(r, u_{\mu}\right)^{2} & =\int_{\Sigma}\left\{\int_{\mathbf{R}^{m}} Q_{k}(r,|y|) P_{k}(\omega \vee y) d \mu(y)\right\}^{2} d \omega \\
& \leqslant \int_{\Sigma}\left\{\int_{\mathbf{R}^{m}}\left|Q_{k}(r,|y|)\right| P_{k}^{2}(\omega \vee y) d|\mu|(y) \int_{\mathbf{R}^{m}}\left|Q_{k}(r,|y|)\right| d|\mu|(y)\right\} d \omega \\
& =\left\{\int_{\Sigma} P_{k}^{2}(\omega \vee e) d \omega\right\}\left\{\int_{0}^{\infty} Q_{k}(r, t) d \mu^{*}(t)\right\}^{2} \\
& =\int_{\Sigma}\left\{\int_{0}^{\infty} Q_{k}(r, t) P_{k}(\omega \vee e) d \mu^{*}(t)\right\}^{2} d \omega=C_{k}\left(r, u_{\mu}^{*}\right)^{2},
\end{aligned}
$$


as claimed. When $k=0$ we have as in (1.9) that

$$
\begin{aligned}
\sigma_{m}^{-1 / 2} C_{0}\left(r, u_{\mu}\right) & =\left|\int_{|y| \leqslant r}[K(r e)-K(y)] d \mu(y)\right| \\
& =|N(r,-u)-N(r, u)| \\
& \leqslant N(r,-u)+N(r, u)=N\left(r,-u_{\mu}^{*}\right)=\sigma_{m}^{-1 / 2} C_{0}\left(r, u_{\mu}^{*}\right) .
\end{aligned}
$$

To prove Theorem $1\left(^{2}\right)$, let $u \in \mathscr{D}_{m}$ have order $\lambda \neq$ positive integer and put $q=[\lambda]$. Then (1.20) holds with $h$ an harmonic polynomial of degree $\leqslant q$ and $u_{\mu} \in \mathscr{D}_{m}$ of order $\lambda$. Further, $N(r)=N_{u}(r)=N\left(r,-u_{\mu}^{*}\right)$ has order $\lambda$ by (1.19); thus there exists a strong proximate order $\lambda(t)$ in the sense of [19, p. 41], that is, $\lambda(t) \in C^{2}(0, \infty)$ and

$$
\lambda(t) \rightarrow \lambda, \quad \lambda^{\prime}(t) t \log t \rightarrow 0, \quad \lambda^{\prime \prime}(t) t^{2} \log t \rightarrow 0 \quad(t \rightarrow \infty),
$$

and, if

$$
N_{1}(t)=t^{\lambda(t)}, \quad n_{1}(t)=d_{m}^{-1} t^{m-1} N_{1}^{\prime}(t)
$$

then also

$$
N(t) \leqslant N_{1}(t) \quad(0<t<\infty), \quad N\left(r_{n}\right)=N_{1}\left(r_{n}\right) \quad(n \geqslant 1)
$$

where $r_{n}$ increases to $+\infty$, and

$$
n_{1}^{\prime}(t)=\left\{\lambda(\lambda+m-2) / d_{m}+o(1)\right\} t^{m-3} N_{1}(t) \quad(t \rightarrow \infty) .
$$

For proof, see pp. 35 and 39 in [19].

In particular, $n_{1}(t)$ is eventually increasing, say for $t \geqslant r_{1}$. Define $\hat{n}, \hat{N}$ by

$$
\begin{aligned}
\hat{N}(t) & =N(t) \quad\left(0<t \leqslant r_{1}\right), \\
& =N_{1}(t) \quad\left(r_{1} \leqslant t<\infty\right) ; \\
\hat{N}(r) & =d_{m} \int_{0}^{r} \hat{n}(t) t^{1-m} d t .
\end{aligned}
$$

Clearly, $\hat{n}$ increases on $(0, \infty)$ and thus

$$
\hat{u}(x)=\int_{0}^{\infty} K_{q}(x, t e) d \hat{n}(t) \in \delta_{m} .
$$

Further,

(2) We thank Dr. F. Abi-Khuzam for pointing out an error in a previous version of the proof of Theorem 1. 
(2.9) $\liminf _{n \rightarrow \infty} \frac{N\left(r_{n}\right)}{m_{2}\left(r_{n}, u\right)} \geqslant \liminf _{n \rightarrow \infty} \frac{N\left(r_{n}\right)}{m_{2}\left(r_{n}, u_{\mu}\right)+m_{2}\left(r_{n}, h\right)} \geqslant \liminf _{n \rightarrow \infty} \frac{N\left(r_{n}\right)}{m_{2}\left(r_{n}, u_{\mu}^{*}\right)}$ where we have used (2.4) and $m_{2}\left(r_{n}, h\right)=O\left(r_{n}^{q}\right)=o\left(N\left(r_{n}\right)\right)$, by (2.6).

We proceed to estimate $m_{2}\left(r_{n}, u_{\mu}^{*}\right)$. For each $k \geqslant 1$, we have from the proof of Theorem 2.1 that

$$
\sigma_{m}^{-1} C_{k}\left(r, u_{\mu}^{*}\right)^{2}=I_{k}^{2}\left\{\int_{0}^{\infty} Q_{k}(r, t) d \mu^{*}(t)\right\}^{2}
$$

where $[11, \mathrm{pp} .15,33,4]$

$$
\begin{aligned}
I_{k}^{2} & =\sigma_{m}^{-1} \int_{\Sigma} P_{k}^{2}(\omega \vee e) d \omega \\
& =\frac{(m-2) \Gamma(k+m-2)}{\Gamma(m-2) \Gamma(k+1)(2 k+m-2)} \quad(k \geqslant 1, m \geqslant 3), \\
I_{k}^{2} & =\frac{1}{2 \pi} \int_{0}^{2 \pi} \frac{\cos ^{2} k \theta}{k^{2}} d \theta=\frac{1}{2 k^{2}} \quad(k \geqslant 1, m=2) .
\end{aligned}
$$

By (1.24), (1.8) and two integrations by parts,

$$
\begin{aligned}
\left|\int_{0}^{\infty} Q_{k}(r, t) d \mu^{*}(t)\right| & =\int_{0}^{\infty}\left|Q_{k}(r, t)\right| d \mu^{*}(t) \\
& =\beta_{k} N(r)+\frac{k(k+m-2)}{d_{m}} \int_{0}^{\infty} N(t)\left|Q_{k}\left(\frac{r}{t}, 1\right)\right| \frac{d t}{t}
\end{aligned}
$$

where

$$
d_{m} \beta_{k}=2 k+m-2 \quad(1 \leqslant k \leqslant q), \quad=-(2 k+m-2) \quad(k>q) .
$$

Thus at the $r_{n}$, by (2.6) and (2.7),

$$
C_{k}\left(r_{n}, u_{\mu}^{*}\right) \leqslant C_{k}\left(r_{n}, \hat{u}\right) \quad(k \geqslant 0) .
$$

It is easy to see from elementary properties of proximate orders that

$$
\liminf _{n \rightarrow \infty} \frac{\hat{N}\left(r_{n}\right)}{m_{2}\left(r_{n}, \hat{u}\right)}=\lim _{r \rightarrow \infty} \frac{\hat{N}(r)}{m_{2}(r, \hat{u})}=K_{2}(\lambda, m),
$$

where

$$
K_{2}(\lambda, m)=r^{\lambda} / m_{2}\left(r, U_{\lambda}\right) \quad(0<r<\infty)
$$

and 


$$
U_{\lambda}(r \omega)=\frac{\lambda(\lambda+m-2)}{d_{m}} \int_{0}^{\infty} K_{q}(r \omega, t e) t^{\lambda+m-3} d t \quad\left(=J_{\lambda}(\omega) r^{\lambda}\right)
$$

is the subharmonic function with $N\left(r,-U_{\lambda}\right) \equiv r^{\lambda}$. (A proof of $(2.11)$ is sketched below.)

For $U_{\lambda}(x)$, clearly

$$
\sigma_{m}^{-1 / 2} C_{k}\left(1, U_{\lambda}\right)=I_{k}\left\{\beta_{k}+\frac{k(k+m-2)}{d_{m}} \int_{0}^{\infty} t^{\lambda}\left|Q_{k}\left(\frac{1}{t}, 1\right)\right| \frac{d t}{t}\right\},
$$

and a direct calculation using (1.24) and

$$
K_{2}(\lambda, m)^{-2}=m_{2}\left(1, U_{\lambda}\right)^{2}=1+\sigma_{m}^{-1} \sum_{k=1}^{\infty} C_{k}\left(1, U_{\lambda}\right)^{2}
$$

shows that $K_{2}(\lambda, m)$ coincides with $C(\lambda, m)$ defined in (14). In view of (2.9) and (2.11), the proof of Theorem 1 (for general $u \in \mathscr{D}_{m}$ ) is complete.

The truth of (2.11) can be seen easily from the integral representation for $\hat{u}(x)$, together with (2.7), (1.14) and properties of proximate orders; we deduce

$$
\lim _{r \rightarrow \infty} \frac{\hat{u}(r \omega)}{r^{\lambda(r)}}=\frac{\lambda(\lambda+m-2)}{d_{m}} \int_{0}^{\infty} K_{q}(\omega, s e) s^{\lambda+m-3} d s
$$

for all $\omega \in \Sigma_{m}, \omega \neq(1,0, \ldots, 0)$. Further, if $\cos \theta=\omega \vee e$ and $\delta>0$ is given, the limit holds uniformly for $\theta$ in $\delta \leqslant|\theta| \leqslant \pi$ and, if $m \leqslant 4$,

$$
\int_{\{\omega:|\theta|<\delta\}}|\hat{u}(r \omega)|^{2} d \omega \leqslant C(\delta) r^{2 \lambda(r)} \quad\left(r \geqslant r_{0}\right)
$$

where $C(\delta) \rightarrow 0$ as $\dot{\delta} \rightarrow 0$; this last can be seen from the estimate

$$
\begin{aligned}
C_{1}|\hat{u}(x)| \leqslant & \int_{2 r}^{\infty}\left(\frac{r}{t}\right)^{q+1} t^{\lambda(t)-1} d t+\int_{r / 2}^{2 r}\left|K_{q}(x, t e)\right| t^{\lambda(t)+m-3} d t \\
& +\int_{r_{1}}^{r / 2}\left(\frac{r}{t}\right)^{q} t^{\lambda(t)-1} d t+\int_{0}^{r_{1}}\left|K_{q}(x, t e)\right| d n(t) \\
& \leqslant r^{\lambda(r)}\left\{C_{2}+2^{\lambda+1} \int_{r / 2}^{2 r}|K(x-t e)| t^{m-3} d t\right\}
\end{aligned}
$$

for all large $r$, where the last integral is of the type considered in (2.5). (An obvious modification is needed in the estimate for $\left[r_{1}, r / 2\right]$ when $m=2, q$ $=0$; cf. Lemma 1.1.)

From

$$
\sum_{k=1}^{\infty} \log \left(1-\frac{\lambda^{2}}{k^{2}}\right)=\log \left(\frac{\sin \pi \lambda}{\pi \lambda}\right)
$$


we observe, after two differentiations with respect to $\lambda$, that

$$
C(\lambda, 2)^{-2}=\frac{1}{2}\left(\frac{\pi \lambda}{\sin \pi \lambda}\right)^{2}\left\{1+\frac{\sin 2 \pi \lambda}{2 \pi \lambda}\right\}
$$

A convenient expression for $C(\lambda, 3)$ is given by

$$
\begin{aligned}
\frac{2 \lambda+1}{\lambda^{2}(\lambda+1)^{2}} C(\lambda, 3)^{-2} & =\sum_{k=-\infty}^{\infty} \frac{1}{(k-\lambda)^{2}}-\sum_{k=1}^{\infty} \frac{1}{(k+\lambda)^{2}}-\sum_{k=0}^{\infty} \frac{1}{(k+\lambda+1)^{2}} \\
& =\left(\frac{\pi}{\sin \pi \lambda}\right)^{2}-2 \sum_{k=1}^{\infty} \frac{1}{(k+\lambda)^{2}},
\end{aligned}
$$

(2.13) $C(\lambda, 3)^{2}=\left(\frac{\sin \pi \lambda}{\pi \lambda}\right)^{2} \frac{2 \lambda+1}{(\lambda+1)^{2}}\left\{1-\frac{2}{\pi^{2}}\left(\sin ^{2} \pi \lambda\right) \sum_{k=1}^{\infty} \frac{1}{(k+\lambda)^{2}}\right\}^{-1}$.

$C(\lambda, 4)$ can be summed explicitly in terms of elementary functions:

$$
\begin{aligned}
\frac{4(\lambda+1)}{\lambda^{2}(\lambda+2)^{2}} C & (\lambda, 4)^{-2} \\
= & \frac{1}{\lambda^{2}}+\sum_{k=1}^{\infty} \frac{k-\lambda+(\lambda+1)}{(k-\lambda)^{2}}-\sum_{k=1}^{\infty} \frac{k+\lambda+1-(\lambda+1)}{(k+\lambda+1)^{2}} \\
= & \frac{1}{\lambda^{2}}+\sum_{k=1}^{\infty}\left\{\frac{1}{k-\lambda}-\frac{1}{k+\lambda+1}\right\} \\
& +(\lambda+1)\left\{\sum_{k=1}^{\infty} \frac{1}{(k-\lambda)^{2}}+\sum_{k=1}^{\infty} \frac{1}{(k+\lambda+1)^{2}}\right\} \\
= & (\lambda+1) \sum_{k=-\infty}^{\infty} \frac{1}{(k-\lambda)^{2}}-\frac{1}{\lambda}-\sum_{k=1}^{\infty}\left\{\frac{1}{\lambda-k}+\frac{1}{\lambda+k}\right\} \\
= & (\lambda+1)\left(\frac{\pi}{\sin \pi \lambda}\right)^{2}-\pi \cot \pi \lambda, \\
C(\lambda, 4)^{2}= & \left(\frac{\sin \pi \lambda}{\pi \lambda}\right)^{2}\left(\frac{2}{\lambda+2}\right)^{2}\left\{1-\frac{\sin 2 \pi \lambda}{2 \pi(\lambda+1)}\right\}^{-1} .
\end{aligned}
$$

We deduce easily

TheOREM 2.2. Let $u \in \mathscr{D}_{m}$ have finite order $\lambda$. Then

$$
\underset{r \rightarrow \infty}{\limsup } \frac{N(r)}{T(r, u)} \geqslant \frac{|\sin \pi \lambda|}{\pi \lambda(\lambda+1)^{\frac{1}{2} m-1}} \quad(0 \leqslant \lambda<\infty ; m \leqslant 4) .
$$


For, by Schwarz's inequality and (1.10),

$$
\begin{aligned}
m_{2}(r, u) & \geqslant \sigma_{m}^{-1} \int_{\Sigma} u(r \omega)^{+} d \omega+\sigma_{m}^{-1} \int_{\Sigma}\{-u(r \omega)\}^{+} d \omega \\
& =T(r, u)-N(r, u)+T(r,-u)-N(r,-u)=2 T(r, u)-N(r)
\end{aligned}
$$

and thus

$$
\frac{k(u)}{2-k(u)}=\limsup _{r \rightarrow \infty} \frac{N(r)}{2 T(r, u)-N(r)} \geqslant C(\lambda, m)
$$

Solving this inequality for $k(u)$ and using simple estimates with (2.12)-(2.14), we obtain (2.15).

3. Bounds for $k(u)$ when $m \geqslant 5$. Theorem 2 is contained in

THEOREM 3.1. Let $u \in \mathscr{D}_{m}$ have finite order $\lambda$. Then

$$
k(u) \geqslant A_{m}|\sin \pi \lambda| /(\lambda+1)^{\frac{1}{2}(m+1)} \quad(0<\lambda<\infty)
$$

where we may take $A_{m}=m^{-m}(m \geqslant 5)$.

We assume $\lambda$ is not a positive integer, and let $q=[\lambda]$. By Lemma 1.2, (1.20) holds with $h$ of degree at most $q$. Then

$$
\begin{aligned}
\sigma_{m}^{-1} \int_{\Sigma}\left|u_{\mu}(r \omega)\right| d \omega & \leqslant \int_{\mathbf{R}^{m}}\left\{\sigma_{m}^{-1} \int_{\Sigma}\left|K_{q}(r \omega, y)\right| d \omega\right\} d|\mu|(y) \\
& =\int_{0}^{\infty} B_{q}(r / t) t^{2-m} d n(t)
\end{aligned}
$$

where

$$
B_{q}(r)=\sigma_{m}^{-1} \int_{\Sigma}\left|K_{q}(r \omega, e)\right| d \omega
$$

here $e$ denotes the unit vector in the positive $x_{1}$-direction.

LEMMA 3.1. When $0<r<\infty$,

$$
B_{q}(r) \leqslant 2 e(m-2)^{\frac{1}{2}(m-2)}(q+1)^{\frac{1}{2}(m-3)} r^{q+1} /(r+1) .
$$

Assuming the validity of (3.1), we put

$$
S(r)=r^{q+1} /(r+1)
$$

and use $r S^{\prime}(r) \leqslant(q+1) S(r)$ to get 


$$
\begin{aligned}
\int_{0}^{\infty} S\left(\frac{r}{t}\right) t^{2-m} d n(t) & =d_{m}^{-1} \int_{0}^{\infty}\left\{d_{m} S\left(\frac{r}{t}\right)+S^{\prime}\left(\frac{r}{t}\right) \frac{r}{t}\right\} d N(t) \\
& \leqslant d_{m}^{-1}(q+m-1)(q+1) \int_{0}^{\infty} S\left(\frac{r}{t}\right) N(t) \frac{d t}{t} .
\end{aligned}
$$

By (2.16), (1.20) and Lemma 3.1,

$$
2 T(r, u) \leqslant N(r)+C_{m}(q) \int_{0}^{\infty} S\left(\frac{r}{t}\right) N(t) \frac{d t}{t}+O\left(r^{q}\right)
$$

where

$$
C_{m}(q)=4 e(m-2)^{\frac{1}{2}(m-2)}(q+1)^{\frac{1}{2}(m+1)} .
$$

For given $\varepsilon>0$, there exists [6, p. 101] a sequence $r_{n} \rightarrow \infty$ with $N(t)$ $\leqslant\left(t / r_{n}\right)^{\lambda-\varepsilon} N\left(r_{n}\right)\left(0<t \leqslant r_{n}\right), N(t) \leqslant\left(t / r_{n}\right)^{\lambda+\varepsilon} N\left(r_{n}\right)\left(t>r_{n}\right)$. Thus

$$
\begin{aligned}
\limsup _{n \rightarrow \infty} \frac{T\left(r_{n}, u\right)}{N\left(r_{n}\right)} & \leqslant \frac{1}{2}\left\{1+C_{m}(q) \int_{0}^{\infty} S(t) t^{-\lambda-1} d t\right\}, \\
k(u) & \geqslant(4 \pi e)^{-1}(m-2)^{\frac{1}{2}(2-m)} \frac{|\sin \pi \lambda|}{(q+1)^{\frac{1}{2}(m+1)}}
\end{aligned}
$$

and Theorem 3.1 follows.

Proof OF LEMMA 3.1. We first suppose $0<r<1$. Then (1.14) implies

$$
K_{q}(r \omega, e)=-\sum_{k=q+1}^{\infty} P_{k}(\omega \vee e) r^{k}
$$

Since the $P_{k}$ are orthogonal on $\Sigma$,

$$
B_{q}(r)^{2} \leqslant \sigma_{m}^{-1} \int_{\Sigma} K_{q}(r \omega, e)^{2} d \omega=\sum_{k=q+1}^{\infty} I_{k}^{2} r^{2 k}
$$

where the $I_{k}$ are given in (2.10). By simple estimates,

$$
I_{k}^{2} \leqslant(m-2)^{2} k^{m-4} \quad(k \geqslant 1) .
$$

Put $r_{0}=\exp \{-1 /(q+1)\}$. Then

$$
B_{q}(r)^{2} \leqslant\left\{e(m-2) r^{q+1}\right\}^{2} \sum_{k=q+1}^{\infty} \psi(k) \quad\left(0 \leqslant r \leqslant r_{0}\right)
$$

where $\psi(x)=x^{p} r_{0}^{2 x}(p=m-4)$ increases on $0<x<x_{0}=(q+1) p / 2$ and then decreases, so that 


$$
\begin{aligned}
& \sum_{k=q+1}^{\infty} \psi(k) \leqslant \int_{q+1}^{\infty} \psi(x) d x+\psi\left(x_{0}\right) \\
&= e^{-2}\left(\frac{q+1}{2}\right)^{p+1}\left\{2^{p}+p 2^{p-1}+p(p-1) 2^{p-2}+\cdots+p !\right\} \\
& \quad+\left(\frac{p(q+1)}{2 e}\right)^{p} \\
&<p^{p}(q+1)^{p+1} \quad(p=m-4), \\
& \quad B_{q}(r) \leqslant e(m-2)^{\frac{1}{2(m-2)}}(q+1)^{\frac{1}{2(m-3)}} r^{q+1} \quad\left(0<r \leqslant r_{0}\right) .
\end{aligned}
$$

For $r>r_{0},(1.13)$ yields

$$
\begin{aligned}
K_{q}(r \omega, e) & =K(r \omega-e)+\sum_{k=0}^{q} P_{k}(\omega \vee e) r^{k}, \\
B_{q}(r) & \leqslant \min \left\{1, r^{2-m}\right\}+\sigma_{m}^{-1} \int_{\Sigma}\left|\sum_{k=0}^{q} P_{k}(\omega \vee e) r^{k}\right| d \omega
\end{aligned}
$$

where the second term is dominated by

$$
Q=\left\{\sigma_{m}^{-1} \int_{\Sigma} \sum_{k=0}^{q}\left(P_{k}(\omega \vee e) r^{k}\right)^{2} d \omega\right\}^{1 / 2}=\left\{\sum_{k=0}^{q} I_{k}^{2} r^{2 k}\right\}^{1 / 2} .
$$

Thus

$$
Q^{2} \leqslant\left(r / r_{0}\right)^{2 q} \sum_{k=0}^{q} I_{k}^{2} \quad\left(r_{0}<r<\infty\right)
$$

and by $(2.10)$

$$
I_{k}^{2} \leqslant \frac{(m-2)^{m-3}}{2 \Gamma(m-2)}(q+1)^{m-4} \quad(1 \leqslant k \leqslant q) .
$$

We deduce

$$
B_{q}(r) \leqslant e(m-2)^{\frac{1}{2}(m-3)}(q+1)^{\frac{1}{(2(m-3)}} r^{q} \quad\left(r_{0}<r<\infty\right),
$$

and (3.1) follows.

4. Examples. For $m \geqslant 3$, let $q<\lambda<q+1$ for some integer $q \geqslant 0$ and consider

$$
U_{\lambda}(x)=\frac{\lambda(\lambda+m-2)}{m-2} \int_{0}^{\infty} K_{q}(x, t e) t^{\lambda+m-3} d t,
$$


a subharmonic function whose Riesz mass is distributed along the positive $x_{1}$ axis with

$$
N(r)=N\left(r,-U_{\lambda}\right)=r^{\lambda} \quad(0<r<\infty)
$$

Then

$$
U_{\lambda}(-x)=\frac{\lambda(\lambda+m-2)}{m-2} I_{\lambda}(\cos \theta) r^{\lambda}
$$

where $x=r \omega, \cos \theta=-\omega \vee e$ and

$$
\begin{aligned}
& I_{\lambda}(\cos \theta)=\int_{0}^{\infty} K_{q}(\tau \omega,-e) \tau^{-\lambda-1} d \tau \\
& =\int_{0}^{\infty}\left\{\sum_{k=0}^{q} P_{k}(\omega \vee e)(-1)^{k} \tau^{-k-m+2}-\frac{1}{\left(1+\tau^{2}+2 \tau \cos \theta\right)^{\nu}}\right\} \tau^{\lambda+m-3} d \tau .
\end{aligned}
$$

Here and below, $\nu=(m-2) / 2$.

We have the representation

$$
I_{\lambda}(\cos \theta)=\frac{1}{e^{2 \pi \lambda i}-1} \int_{\Gamma} \frac{z^{\lambda+m-3} d z}{\left(1+z^{2}+2 z \cos \theta\right)^{\nu}},
$$

where $\Gamma$ consists of the circles $|z|=R$ and $|z|=\varepsilon(0<\varepsilon<1<R)$ respectively oriented positively and negatively, joined by segments along the upper and lower edges of the real axis between $\varepsilon$ and $R$. To see this, use

$$
\left(1+z^{2}+2 z \cos \theta\right)^{-\nu}=\sum_{k=0}^{\infty}(-1)^{k} P_{k}(\cos \theta) z^{-k-m+2} \quad(|z|=R)
$$

in (4.4) with Cauchy's theorem and let $\varepsilon \rightarrow 0, R \rightarrow \infty$. Thus we can evaluate $I_{\lambda}$ by residues when $m$ is even. (This procedure is used by Hayman [8, Chapter 4] for orders $\lambda<1$.)

We deduce

$$
I_{\lambda}(\cos \theta)=\frac{2 \pi i}{e^{2 \pi \lambda i}-1}\left\{\frac{g^{(\nu-1)}(\bar{a})}{(\nu-1) !}+\frac{\bar{g}^{(\nu-1)}(a)}{(\nu-1) !}\right\}
$$

where $g(z)=z^{\lambda+m-3}(z-a)^{-\nu}, \bar{g}$ is the similar expression with $\bar{a}$ in place of $a$, and $a=-e^{i \theta}$. By direct calculation,

$$
I_{\lambda}(\cos \theta)=\frac{\pi}{\sin \pi \lambda} \frac{\sin (\lambda+1) \theta}{\sin \theta} \quad(\nu=1)
$$

and for $\nu>1$, 
$I_{\lambda}(\cos \theta)$

$$
\begin{aligned}
=\frac{\pi}{\sin \pi \lambda}\left\{\frac{(\lambda+m-3) \cdots(\lambda+m-\nu-1)}{2^{\nu-1}(\nu-1) !}\right. \\
\left.\cdot \frac{\cos [(\lambda+\nu) \theta-\pi \nu / 4]}{(\sin \theta)^{\nu}}+R\right\}
\end{aligned}
$$

where

$$
|R| \leqslant C(\nu)(\lambda+1)^{\nu-2}(\sin \theta)^{3-m} \quad(0<\theta<\pi)
$$

and $C(\nu)$ does not depend on $\theta$ or $\lambda$. This follows easily from (4.5) and

$$
g^{(\nu-1)}(z)=\sum_{j=0}^{\nu-1}\left(\begin{array}{c}
\nu-1 \\
j
\end{array}\right) D^{(\nu-j-1)}\left(z^{\lambda+m-3}\right) D^{(j)}\left((z-\bar{a})^{-\nu}\right)
$$

where $D=d / d z$, and the similar expression for $\bar{g}^{(\nu-1)}$.

Since $I_{\lambda}(\cos \theta)$ is even in $\theta$,

$$
r^{-\lambda} T\left(r, U_{\lambda}\right)=\frac{\lambda(\lambda+m-2)}{m-2} 2 \sigma_{m}^{-1} \int_{0}^{\pi} I_{\lambda}(\cos \theta)^{+} d \omega(\theta) \equiv K(\lambda, m)^{-1}
$$

where

$$
d \omega(\theta)=\sigma_{m-1}(\sin \theta)^{m-2} d \theta
$$

Thus

$$
T\left(1, U_{\lambda}\right)=\frac{\pi \lambda}{|\sin \pi \lambda|} \frac{(\lambda+m-2) \cdots(\lambda+m-\nu-1)}{\nu ! 2^{\nu-1}}\left(\frac{\sigma_{m-1}}{\sigma_{m}}\right) H_{\lambda}
$$

where

$$
H_{\lambda}=\int_{0}^{\pi}\left\{(-1)^{q} \cos \left[(\lambda+\nu) \theta-\frac{\pi \nu}{4}\right](\sin \theta)^{\nu}\right\} d \theta+\varepsilon_{\lambda},
$$

with $\left|\varepsilon_{\lambda}\right| \leqslant C_{1}(\nu) /(\lambda+1)$ by (4.7). On the other hand, since

$$
\begin{aligned}
\lim _{\beta \rightarrow \infty} \int_{a}^{b} f(\theta) \stackrel{+}{\cos }(\beta \theta+\gamma) d \theta \\
\quad=\lim _{\beta \rightarrow \infty} \int_{a}^{b} f(\theta)\{\cos (\beta \theta+\gamma)\}^{-} d \theta=\frac{1}{\pi} \int_{a}^{b} f(\theta) d \theta
\end{aligned}
$$

for any $f \in L^{1}(a, b)$ and $\gamma$ real, we obtain

$$
H_{\lambda}=\frac{1}{\pi} \int_{0}^{\pi} \sin ^{\nu} \theta d \theta+o(1)
$$


on letting $\lambda \rightarrow \infty$ so that first $q=[\lambda]$ is even, then odd.

We deduce that the $U_{\lambda}$ satisfy

$$
\begin{aligned}
\frac{N(r)}{T\left(r, U_{\lambda}\right)} & \equiv \frac{\sigma_{m}(m-2)}{2 \lambda(\lambda+m-2)}\left\{\int_{0}^{\pi} I_{\lambda}(\cos \theta)^{+} d \omega(\theta)\right\}^{-1} \\
& =\alpha_{m}|\sin \pi \lambda| \lambda^{-\frac{1}{2} m}\{1+o(1)\} \quad(0<r<\infty ; \lambda \rightarrow \infty)
\end{aligned}
$$

where $\alpha_{m}$ depends only on the dimension; this proves (16) for $m$ even.

In fact, from (4.4) $I_{\lambda}(\cos \theta)$ can be seen to satisfy a differential equation of hypergeometric type [17, p. 178], thus [17, pp. 175, 104]

$$
\begin{aligned}
I_{\lambda}(\cos \theta) & =\beta_{2} F_{1}\left(\lambda+2 \nu,-\lambda ; \nu+\frac{1}{2} ; \frac{1+\cos \theta}{2}\right), \\
\beta & =I_{\lambda}(1) \Gamma\left(\frac{1}{2}+\nu+\lambda\right) \Gamma\left(\frac{1}{2}-\nu-\lambda\right) / \Gamma\left(\frac{1}{2}+\nu\right) \Gamma\left(\frac{1}{2}-\nu\right)
\end{aligned}
$$

where the ${ }_{2} F_{1}$ has a known asymptotic expansion [17, p. 77] for large $\lambda$ like that in (4.6), but valid for all real $\nu$. Further, our analysis giving (4.6) from (4.4), when $\nu$ is integral, remains valid for half-integral $\nu$ in the case $\theta=0$, and we can asymptotically evaluate the factor $I_{\lambda}(1)$ in (4.9). (The ${ }_{2} F_{1}$ in (4.9) is essentially a Gegenbauer function $[17$, p. 175].)

We conclude that the functions $U_{\lambda}$ satisfy (16) for any $m \geqslant 3$, by known asymptotic results. When $m=2$, (4.1) gives $U_{\lambda}(x)=\pi \lambda \csc \pi \lambda(\cos \theta \lambda) r^{\lambda}$ for all $\lambda \neq$ positive integer, $|\theta| \leqslant \pi, r>0$.

5. Proof of Theorem 3. We can assume all the Riesz mass of $u(x)$ is on the negative $x_{1}$-axis, so that

$$
u(x)=\int_{0}^{\infty} K_{q}(x,-t e) d \mu(t)+h(x)=u_{\mu}(x)+h(x)
$$

where $u$ has order $\lambda \in(q, q+1)$ and the degree of $h(x)$ is at most $q$. For any $\gamma \in(\lambda, q+1)$,

$$
\begin{array}{r}
\int_{0}^{\infty} u_{\mu}(r \omega) r^{-\gamma-1} d r=\int_{0}^{\infty} d \mu(t) \int_{0}^{\infty} K_{q}(r \omega,-t e) r^{-\gamma-1} d r \\
=\int_{0}^{\infty} t^{-\gamma-m+2} d \mu(t) \int_{0}^{\infty} K_{q}(\tau \omega,-e) \tau^{-\gamma-1} d \tau \\
=\frac{\gamma(\gamma+m-2)}{m-2} I_{\gamma}(\cos \theta) \int_{0}^{\infty} N(t) t^{-\gamma-1} d t
\end{array}
$$

where $I_{\gamma}$ is defined in (4.3).

Let $\mathscr{E} \subset \Sigma \cap\left\{x_{m} \geqslant 0\right\}$ be measurable $d \omega$, and define $E \subset[0, \pi]$ by $E$ $=\{\theta: \omega \vee e=\cos \theta, \omega \in \mathcal{E}\}$, and 


$$
T\left(r, u_{\mu} ; \mathscr{E}\right)=2 \sigma_{m}^{-1} \int_{\mathscr{E}} u_{\mu}(r \omega) d \omega
$$

Thus $T\left(r, u_{\mu} ; \varepsilon\right) \leqslant T\left(r, u_{\mu}\right)$ and by $(5.2)$

$$
\begin{aligned}
\int_{0}^{\infty} T\left(r, u_{\mu} ; \mathcal{E}\right) & r^{-\gamma-1} d r \\
& =\frac{\gamma(\gamma+m-2)}{m-2}\left\{2 \sigma_{m}^{-1} \int_{E} I_{\gamma}(\cos \theta) d \omega(\theta)\right\} \int_{0}^{\infty} N(t) t^{-\gamma-1} d t
\end{aligned}
$$

where $d \omega(\theta)$ was defined in $\S 4$.

Using a theorem of Pólya [13] just as in [9, pp. 225-227], we deduce

$$
\liminf _{r \rightarrow \infty} \frac{A(\lambda) N(r)+r^{\tau}}{T\left(r, u_{\mu}\right)} \leqslant 1
$$

where $\tau<\lambda$ is arbitrary and

$$
A(\gamma)=\frac{\gamma(\gamma+m-2)}{m-2} 2 \sigma_{m}^{-1} \int_{E} I_{\gamma}(\cos \theta) d \omega(\theta) .
$$

Since $N(r) \leqslant T\left(r, u_{\mu}\right)$, it follows from (5.3) that there exists $\left\{r_{n}\right\} \rightarrow \infty$ with

$$
A(\lambda) \liminf _{n \rightarrow \infty} \frac{N\left(r_{n}\right)}{T\left(r_{n}, u_{\mu}\right)} \leqslant 1
$$

and

$$
\lim _{n \rightarrow \infty} \frac{\log T\left(r_{n}, u_{\mu}\right)}{\log r_{n}}=\lambda
$$

Thus by (5.1)

$$
A(\lambda) \liminf _{r \rightarrow \infty} \frac{N(r)}{T(r, u)} \leqslant 1 .
$$

Since $E$ is an arbitrary subset of $[0, \pi]$ and $I_{\gamma}$ is independent of $r$, we can take

$$
E=\left\{\theta: I_{\lambda}(\cos \theta) \geqslant 0\right\} .
$$

Then by (4.8) and (5.4), (18) follows. Assertion (19) is a simple consequence of

$$
\lim _{\theta \rightarrow \pi^{-}} I_{\lambda}(\cos \theta)=-\infty \quad(m \geqslant 3),
$$

clear from (4.3). When $m$ is even, $K(\lambda, m)$ can be computed in terms of elementary functions; in particular, (20) follows from the evaluation $I_{\lambda}(\cos \theta)$ $=(\pi / \lambda \sin \pi \lambda) \cos \theta \lambda$ when $m=2$. 


\section{REFERENCES}

1. W. Donoghue, Distributions and Fourier transforms, Academic Press, New York, 1969.

2. A. Edrei and W. H. J. Fuchs, On the growth of meromorphic functions with several deficient values, Trans. Amer. Math. Soc. 93 (1959), 292-328. MR 22 \#770.

3. - The deficiencies of meromorphic functions of order less than one, Duke Math. J. 27 (1960), 233-249. MR 23 \#A1039.

4. A. Edrei, W. H. J. Fuchs and S. Hellerstein, Radial distribution and deficiencies of the values of a meromorphic function, Pacific J. Math. 11 (1961), 135-151. MR 23 \# A330.

5. A. A. Gol'dberg and I. V. Ostrovskii,, The distribution of values of meromorphic functions, Izdat. "Nauka", Moscow, 1970. MR 43 \#6439.

6. W. K. Hayman, Meromorphic functions, Clarenden Press, Oxford, 1964. MR 29 \# 1337.

7. - Subharmonic functions in $R^{m}$, Actes, Congrès Internat. Math. (Nice, 1970), Vol. 2, Gauthier-Villars, Paris, 1971, pp. 601-605.

8. W. K. Hayman and P. B. Kennedy, Subharmonic functions, vol. 1, Academic Press, New York, 1976.

9. S. Hellerstein and D. F. Shea, Bounds for the deficiencies of meromorphic functions of finite order, Proc. Sympos. Pure Math., vol. 11, Amer. Math. Soc., Providence, R.I., 1968, pp. 214-239. MR 38 \# 4684.

10. J. Miles and D. F. Shea, An extremal problem in value-distribution theory, Quart. J. Math. Oxford Ser. (2) 24 (1973), 377-383. MR 48 \#2393.

11. C. Müller, Spherical harmonics, Lecture Notes in Math., vol. 17, Springer-Verlag, Berlin and New York, 1966. MR 33 \#7593.

12. I. V. Ostrovskiī, Certain asymptotic properties of entire functions with real negative zeros, Zap. Meh.-Mat. Fak. i Har'kov. Mat. Obšč. (4) 28 (1961), 23-32. MR 40 \#2863.

13. G. Pólya, On the minimum modulus of integral functions of order less than unity, J. London Math. Soc. 1 (1926), 78-86.

14. T. Radó, Subharmonic functions, Ergebnisse Math. Grenz., Band 5, Springer-Verlag, Berlin, 1937.

15. D. F. Shea, On the Valiron deficiencies of meromorphic functions of finite order, Trans. Amer. Math. Soc. 124 (1966), 201-227. MR 34 \#351.

16. E. T. Whittaker and G. N. Watson, $A$ course of modern analysis, Cambridge Univ. Press, London and New York, 1927.

17. A. Erdélyi, W. Magnus, F. Oberhettinger, and F. G. Tricomi, Higher transcendental functions, vol. I, McGraw-Hill, New York, 1953. MR 15, 419.

18. M. G. Arsove, Functions representable as differences of subharmonic functions, Trans. Amer. Math. Soc. 75 (1953), 327-365. MR 15, 526.

19. B. Ja. Levin, Distribution of zeros of entire functions, GITTL, Moscow, 1956; English transl., Transl. Math. Monographs, vol. 5, Amer. Math. Soc., Providence, R.I., 1964. MR 19, 402; 28 \#217.

Department of Mathematics, Tata Institute of Fundamental Research, Bombay, India

Department of Mathematics, University of Toledo, Toledo, Ohio 43606 (Current address of N. V. Rao)

Department of Mathematics, University of Wisconsin, Madison, Wisconsin 53706 (Current address of D. F. Shea) 